The proofs for this theorem and the next two are very similar to the proofs of the first three and hence will not be given. The cases where a cusp falls on the lines $x_{i}=0$ may easily be recognized. Thus if any two of $t_{1}, t_{2}, t_{3}$ are equal, a cusp falls at a vertex of the triangle of reference, and if the corresponding pair from $r_{1}, r_{2}, r_{3}$ are equal, $B$ vanishes. A cusp on the lines $x_{1}=0$ but not at a vertex may be found by noting that $t_{1}, t_{2}, t_{3}$ are the only possible parameters for such a cusp and that the tangent line through it is indeterminate. In this case also $B$ may vanish.

TheORem 5. If the cubic (3) is cuspidal, $B$ may or may not be zero when the cusp is on one of the lines $x_{i}=0,(i=1,2,3)$, and $B$ is different from zero for all other cases.

THEOREM 6. A triangle of reference, circumscribed to any nodal cubic, can always be chosen such that $B$ vanishes.

The University of California at Los Angeles

\title{
AN APPLICATION OF METRIC GEOMETRY TO DETERMINANTS*
}

BY L. M. BLUMENTHAL

1. Introduction. A paper presented to the Accademia dei Lincei by B. Segre $\dagger$ is devoted to the following theorem, announced by $\mathrm{H}$. W. Richmond :

If in a non-vanishing, symmetric determinant of order six, the six elements in the principal diagonal are all zero, and the complementary minors of five of these elements are also zero, then the complementary minor of the remaining element must be zero.

Segre states that the analogous theorem for determinants of the second $\S$ and the fourth orders may be immediately verified, and the object of his investigation is to ascertain if analogous theorems are valid for determinants of other orders. He shows

* Presented to the Society, September 9, 1931.

$\dagger$ Intorno ad una proprietd dei determinanti simmetrici del $6^{\circ}$ ordine, Atti dei Lincei, (6), vol. 2 (1925), p. 539.

$\ddagger$ On the property of a double-six of lines, and its meaning in hypergeometry, Proceedings of the Cambridge Philosophical Society, vol. 14 (1908), p. 475. The statement of the theorem given in this paper contains no explicit hypothesis relative to the non-vanishing of the determinant.

$\S$ The theorem is, of course, trivial for second-order determinants. 
that such theorems may possibly hold for determinants of the eighth order, and that this is the only possible additional extension of Richmond's theorem.

The purpose of this note is to show by means of examples that, far from being readily verified for determinants of order 4 , the analogous theorem is not true for fourth-order determinants. It follows immediately that the theorem announced is not true for determinants of order six, nor the analogous theorem for determinants of order eight.

The emphasis of this note, however, is laid upon a "countertheorem"* suggested by considerations in metric geometry (in the sense of Karl Menger).

2. Failure of Richmond's Theorem. Consider the symmetric determinant

$$
D_{4}=\left|\begin{array}{llll}
0 & 1 & 0 & 0 \\
1 & 0 & 1 & 1 \\
0 & 1 & 0 & 1 \\
0 & 1 & 1 & 0
\end{array}\right|=1
$$

The complementary minor of the element in the first row and first column has the value 2 , whereas the complementary minors of the other three elements in the principal diagonal are all zero. Hence $D_{4}$ is a fourth-order determinant satisfying the hypotheses of Richmond's theorem stated for fourth-order determinants, and for which the conclusion of the theorem does not hold.

To obtain a sixth-order determinant for which the theorem is false, it is only necessary to border the determinant $D_{4}$ obtaining

$$
D_{6}=\left|\begin{array}{llllll}
0 & 1 & 0 & 0 & 0 & 0 \\
1 & 0 & 1 & 1 & 0 & 0 \\
0 & 1 & 0 & 1 & 0 & 0 \\
0 & 1 & 1 & 0 & 0 & 0 \\
0 & 0 & 0 & 0 & 0 & 1 \\
0 & 0 & 0 & 0 & 1 & 0
\end{array}\right| .
$$

* This theorem was suggested by Karl Menger, to whom I am indebted for valuable counsel in preparing this note. 
Then $D_{6}=-D_{4}=-1$, and the only element in the principal diagonal that has a non-vanishing complementary minor is the element in the first row and the first column. It is clear that a determinant of order eight that violates the theorem stated for determinants of this order can be formed from $D_{6}$ in a manner similar to the way in which $D_{6}$ was formed from $D_{4}$.

3. The Counter Theorem. Segre shows that determinants of order 5 may be constructed for which the analogous theorem is not valid. He exhibits also a fifth-order determinant that does satisfy such a theorem. We shall prove the following theorem.

THEOREM. If the symmetric determinant

$$
D=\left|\begin{array}{lllll}
0 & 1 & 1 & 1 & 1 \\
1 & 0 & r_{12} & r_{13} & r_{14} \\
1 & r_{21} & 0 & r_{23} & r_{24} \\
1 & r_{31} & r_{32} & 0 & r_{34} \\
1 & r_{41} & r_{42} & r_{43} & 0
\end{array}\right|, \quad \quad\left(r_{i j}=r_{j i}\right)
$$

with $r_{i j}>0,(i, j=1,2,3,4), i \neq j$, is different from zero, and the complementary minors of four of the elements in the principal diagonal vanish, then the complementary minor of the remaining element does not vanish.

Since the elements $r_{i j}$ are all positive, we may set $r_{i j}=(i j)^{2}$, where we interpret $(i j)^{2}$ as the square of the distance between a point $p_{i}$ and a point $p_{j}$ of a quadruple $p_{1}, p_{2}, p_{3}, p_{4}$. Suppose now that the conditions of the theorem are satisfied. Then there are, a priori, two cases possible.

CASE A. The four bordered complementary minors all vanish. We show then that the fifth minor

$$
\mathcal{E}\left(p_{1}, p_{2}, p_{3}, p_{4}\right)=\left|\begin{array}{cccc}
0 & (12)^{2} & (13)^{2} & (14)^{2} \\
(21)^{2} & 0 & (23)^{2} & (24)^{2} \\
(31)^{2} & (32)^{2} & 0 & (34)^{2} \\
(41)^{2} & (42)^{2} & (43)^{2} & 0
\end{array}\right|
$$

does not vanish.

Now the vanishing of a bordered minor (a bordered determi- 
nant of three points) means, according to the Heronian formula,* that one of the points is between $\dagger$ the two others; that is, that the three points are linear. Hence for Case A all four of the triples that are selected from the four points $p_{1}, p_{2}, p_{3}, p_{4}$ are linear. On the other hand, the determinant $D$ is not zero. This implies that the four points $p_{1}, p_{2}, p_{3}, p_{4}$ are not linear, but form a pseudo-linear quadruple.f Then the following relations subsist:§

$(12)=(34)=a$

$(23)=(14)=b$,

$(31)=(24)=a+b$;

and hence we may write

$$
\mathcal{E}\left(p_{1}, p_{2}, p_{3}, p_{4}\right)=\left|\begin{array}{cccc}
0 & a^{2} & (a+b)^{2} & b^{2} \\
a^{2} & 0 & b^{2} & (a+b)^{2} \\
(a+b)^{2} & b^{2} & 0 & a^{2} \\
b^{2} & (a+b)^{2} & a^{2} & 0
\end{array}\right| .
$$

To show this determinant to be different from zero, we consider the more general symmetric determinant

$$
\Delta=\left|\begin{array}{llll}
0 & \alpha^{2} & \beta^{2} & \gamma^{2} \\
\alpha^{2} & 0 & \delta^{2} & \epsilon^{2} \\
\beta^{2} & \delta^{2} & 0 & \zeta^{2} \\
\gamma^{2} & \epsilon^{2} & \zeta^{2} & 0
\end{array}\right|
$$

If each row of this determinant be multiplied by the product of those three of the six elements not contained in the row, and each column of the determinant be divided by the product of the three elements contained in the column, it is found that

* Thus:

$$
\left|\begin{array}{cccc}
0 & 1 & 1 & 1 \\
1 & 0 & (12)^{2} & (13)^{2} \\
1 & (21)^{2} & 0 & (23)^{2} \\
1 & (31)^{2} & (32)^{2} & 0
\end{array}\right|=[(12)+(23)+(31)][(12)+(23)-(31)]
$$

$\dagger$ A point $p_{2}$ is said to lie between two points $p_{1}, p_{3}$ if $p_{1} p_{2}+p_{2} p_{3}=p_{1} p_{3}$.

$\ddagger$ The proof of the existence of such sets of four points, and their characterization is due to Karl Menger, Untersuchungen über allgemeine Metrik, Mathematische Annalen, vol. 100 (1928), p. 125.

$\S$ Menger, loc. cit., p. 127; see also Mathematische Zeitschrift, vol. 33, p. 408. 


$$
\Delta=\left|\begin{array}{llll}
0 & \alpha \zeta & \beta \epsilon & \gamma \delta \\
\alpha \zeta & 0 & \gamma \delta & \beta \epsilon \\
\beta \epsilon & \gamma \delta & 0 & \alpha \zeta \\
\gamma \delta & \beta \epsilon & \alpha \zeta & 0
\end{array}\right|
$$

from which $\Delta$ is readily expressible in the factored form as $\Delta=(\alpha \zeta+\beta \epsilon+\gamma \delta)(\alpha \zeta+\beta \epsilon-\gamma \delta)(\alpha \zeta-\beta \epsilon+\gamma \delta)(\alpha \zeta-\beta \epsilon-\gamma \delta)$.

Turning now to the determinant $\mathcal{E}\left(p_{1}, p_{2}, p_{3}, p_{4}\right)$ and substituting $\alpha=\zeta=a ; \beta=\epsilon=\alpha+b ; \gamma=\delta=b$ in the expression for $\Delta$, we find that

$$
\mathcal{E}\left(p_{1}, p_{2}, p_{3}, p_{4}\right)=16 a^{2} b^{2}(a+b)^{2}\left(a^{2}+a b+b^{2}\right)
$$

and hence $\mathcal{E}$ is not zero.

CASE B. Three of the four bordered minors are zero, and $\mathcal{E}\left(p_{1}, p_{2}, p_{3}, p_{4}\right)$ is zero. We shall show that Case B is impossible.

From the hypothesis that three of the four bordered minors vanish, it follows that for three of the four triples $p_{i_{1}}, p_{i_{2}}, p_{i_{3}}$, one point lies between the two others. Then only one of the two following cases is possible.*

Sub-CASE $\alpha$. One point, let us say $p_{1}$, lies between each two of the three others. Since $\mathcal{E}\left(p_{1}, p_{2}, p_{3}, p_{4}\right)$ vanishes, the four points do not form a pseudo-linear quadruple, and as $D$ does not vanish, the four points are not linear. Hence the three points $p_{2}, p_{3}, p_{4}$ are not linear, and the following relations exist:

$$
\begin{array}{lll}
(12)=a, & (13)=b, & (14)=c \\
(23)=a+b, & (24)=a+c, & (34)=b+c .
\end{array}
$$

The determinant $D\left(p_{1}, p_{2}, p_{3}, p_{4}\right)$ now has the form

$$
\left|\begin{array}{ccccc}
0 & 1 & 1 & 1 & 1 \\
1 & 0 & a^{2} & b^{2} & c^{2} \\
1 & a^{2} & 0 & (a+b)^{2} & (a+c)^{2} \\
1 & b^{2} & (a+b)^{2} & 0 & (b+c)^{2} \\
1 & c^{2} & (a+c)^{2} & (b+c)^{2} & 0
\end{array}\right|
$$

* Menger, loc. cit., p. 107. 
and substituting again in $\Delta$

$$
\alpha=a, \beta=b, \gamma=c, \delta=a+b, \epsilon=a+c, \zeta=b+c,
$$

we find

$$
\varepsilon\left(p_{1}, p_{2}, p_{3}, p_{4}\right)=-16 a^{2} b^{2} c^{2}(a b+b c+c a) \neq 0,
$$

which contradicts the hypothesis

$$
\varepsilon\left(p_{1}, p_{2}, p_{3}, p_{4}\right)=0 \text {. }
$$

Hence this sub-case is impossible.

Sub-Case $\beta$. The relations $p_{2}$ lies between $p_{1}$ and $p_{3} ; p_{3}$ lies between $p_{2}$ and $p_{4} ; p_{4}$ lies between $p_{3}$ and $p_{1}$ subsist; that is, we may write

$$
\begin{aligned}
& p_{1} p_{2}+p_{2} p_{3}=p_{1} p_{3}, \\
& p_{2} p_{3}+p_{3} p_{4}=p_{2} p_{4}, \\
& p_{3} p_{4}+p_{4} p_{1}=p_{3} p_{1} .
\end{aligned}
$$

Then we have

$$
\begin{array}{lll}
(12)=a, & (13)=a+b, & (14)=a+b-c, \\
(23)=b, & (24)=b+c, & (34)=c ;
\end{array}
$$

and the determinant $D$ takes the form

$$
\left|\begin{array}{ccccc}
0 & 1 & 1 & 1 & 1 \\
1 & 0 & a^{2} & (a+b)^{2} & (a+b-c)^{2} \\
1 & a^{2} & 0 & b^{2} & (b+c)^{2} \\
1 & (a+b)^{2} & b^{2} & 0 & c^{2} \\
1 & (a+b-c)^{2} & (b+c)^{2} & c^{2} & 0
\end{array}\right| .
$$

From this, the determinant $\varepsilon\left(p_{1}, p_{2}, p_{3}, p_{4}\right)$ is found to have the value

$$
\mathcal{E}\left(p_{1}, p_{2}, p_{3}, p_{4}\right)=16 b^{2} c^{2}(a+b)^{2}\left(a b+a c+b^{2}\right) \neq 0,
$$

which again contradicts the hypothesis that $\varepsilon\left(p_{1}, p_{2}, p_{3}, p_{4}\right)$ vanishes. Thus this sub-case has been shown to be impossible, and Case B cannot exist. The theorem is then proved.

Since we have shown that for a determinant satisfying the conditions of the theorem the case $B$ is impossible, it follows that Case A alone is valid. We have, then, the following corollaries: 
Corollary 1. A determinant satisfying the conditions of the theorem is necessarily of the form

$$
\left|\begin{array}{lllll}
0 & 1 & 1 & 1 & 1 \\
1 & 0 & a^{2} & b^{2} & c^{2} \\
1 & a^{2} & 0 & c^{2} & b^{2} \\
1 & b^{2} & c^{2} & 0 & a^{2} \\
1 & c^{2} & b^{2} & a^{2} & 0
\end{array}\right|
$$

where one of the three numbers $a, b, c$ is the sum of the two others.

COROLlaRY 2. There is a double infinity of determinants that satisfy the conditions of the theorem.

COROLlARY 3. If a determinant satisfies the conditions of the theorem, then necessarily the unbordered fourth-order principal minor is different from zero.

COROLLARY 4. If $D$ is a determinant satisfying the conditions of the theorem, and $\mathcal{E}$ is the unbordered fourth-order principal minor of $D$, then there exist three positive numbers $a, b, c$, one of which is the sum of the other two, such that

$$
D=-32 a^{2} b^{2} c^{2}, \quad \varepsilon=-\frac{1}{2}\left(a^{2}+a b+b^{2}\right) D .
$$

In conclusion, the theorem with which this note is concerned may be formulated in the following manner:

If the symmetric determinant

$$
D=\left|\begin{array}{lllll}
0 & 1 & 1 & 1 & 1 \\
1 & 0 & r_{12} & r_{13} & r_{14} \\
1 & r_{21} & 0 & r_{23} & r_{24} \\
1 & r_{31} & r_{32} & 0 & r_{34} \\
1 & r_{41} & r_{42} & r_{43} & 0
\end{array}\right|, \quad\left(r_{i j}=r_{j i}>0, i \neq j\right)
$$

has all five of the complementary minors of the five elements in the principal diagonal zero, then the determinant vanishes.

The Rice Institute 